Reprod. Nutr. Dévelop., 1988, 28 (1), 147-148.

\title{
Comparison in vivo et in vitro (Rusitec) de l'effet d'un antibiotique ionophore sur quelques paramètres fermentaires du rumen
}

Catherine BOGAERT, L. GOMEZ $\left({ }^{*}\right)$, J. P. JOUANY $\left({ }^{*}\right)$

Université de Clermont II, Laboratoire de Chimie Organique Biologique, Les Cézeaux, 63170 Aubière, France.

(*) Laboratoire de la Digestion des Ruminants, I.N.R.A., Theix, 63122 Ceyrat, France.

Summary. Sheep were adapted to a hay: concentrate $(70: 30)$ diet containing no or $33 \mathrm{ppm}$ of an ionophore antibiotic (lasalocid). The influence of this antibiotic on fermentations $\left(\mathrm{pH}, \mathrm{NH}_{3}-\mathrm{N}, \mathrm{VFA}\right)$ was measured in vivo and compared with an in vitro system (Rusitec) inoculated with rumen contents from the same animals. Changes observed were qualitatively the same, but they were quantitatively larger in Rusitec than in sheep.

Le Rusitec est un dispositif semi-continu utilisé pour simuler les processus fermentaires du rumen. Si son emploi s'est développé ces dix dernières années, peu d'études ont été faites parallèlement in vivo, de façon à cerner les limites de ce système in vitro. L'emploi d'antibiotiques ionophores, molécules qui modifient profondément les paramètres fermentaires dans le rumen (revue de Durand, 1982), a semblé approprié pour vérifier l'efficacité de la simulation.

Matériel et méthodes. Quatre moutons adultes, porteurs de canules au niveau du rumen et répartis en 2 lots, reçoivent en alimentation continue (toutes les $3 \mathrm{~h}$ ), 2 régimes présentés sous forme agglomérée $(68 \%$ de foin de fétuque, $12 \%$ de tourteau de soja, $18 \%$ de manioc et $2 \%$ d'un complément minéral vitaminique ; $1100 \mathrm{~g} / \mathrm{j})$, avec de la paille hachée $(100 \mathrm{~g} / \mathrm{j})$, selon un schéma en carré latin. Le premier régime est distribué sans additif ; l'autre contient $33 \mathrm{ppm}$ de lasalocide. Sur du contenu de rumen prélevé à trois reprises sur chaque animal $1 \mathrm{~h}$ après l'ingestion d'aliment, nous avons mesuré le $\mathrm{pH}$ et dosé l'azote ammoniacal (N$\mathrm{NH}_{3}$ ) et les acides gras volatils (AGV). En outre, dans chaque lot, un inoculum a été constitué à partir de contenus de rumen prélevés au cours de la dernière période, pour ensemencer un fermenteur semi-continu de type Rusitec (Czerkawski et Breckenridge, 1977), recevant chaque jour $16 \mathrm{~g}$ (en MS) de l'aliment correspondant à l'inoculum. Pendant 14 jours, nous avons mesuré les paramètres fermentaires $\left(\mathrm{pH}, \mathrm{AGV}, \mathrm{N}-\mathrm{NH}_{3}\right)$ sur les effluents liquides (recueillis pendant $24 \mathrm{~h}$ ) de chaque fermenteur. Les rêsultats obtenus in vivo (sur un milieu fermentaire stabilisé par l'alimentation continue) et in vitro (sur les effluents moyens collectés en 24 h) ont été traités par analyse de variance.

Résultats et discussion. La plupart des paramètres fermentaires mesurés ont été modifiés dans le même sens chez les animaux et dans le Rusitec (tabl. 1). Ainsi, l'effet positif du lasalocide sur la proportion d'acide propionique (C3) dans le mélange des $A G V$ au détriment de l'acide acétique (C2) et surtout de l'acide butyrique (C4), est apparu nettement dans les deux milieux. On note toutefois que les proportions des acides valériques, linéaire (C5) et ramifié (IC5), sont accrues seulement in vitro en présence de lasalocide. 
Globalement, I'absence d'action de la molécule testée sur la concentration totale d'AGV apparaît à la fois in vitro et in vivo. La diminution faible mais significative de la valeur du $\mathrm{pH}$ dans les fermenteurs recevant l'antibiotique est difficile à expliquer.

TABL. 1. - Variation comparée in vivo et in vitro de quelques paramètres fermentaires en présence de lasalocide (L) par rapport au témoin (T).

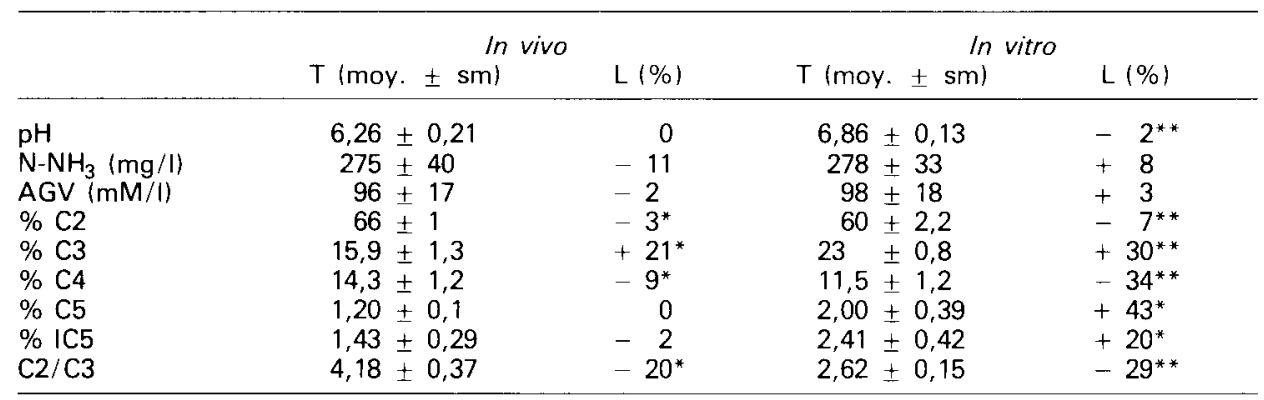

Les signes * et * indiquent un effet « lasalocide " significatif aux seuils respectifs de : $P<0,05$ et $P$ $<0,01$.

Nous avons généralement observé que l'effet antibiotique était plus important in vitro que in vivo bien que les fermenteurs aient été ensemencés à partir d'animaux adaptés aux régimes expérimentaux. Un rapport phase solide/phase liquide faible en fermenteur $(0,02)$ par rapport au rumen $(0,15)$, associé à des modifications de l'équilibre microbien (diminution de certains protozoaires et certaines bactéries), pourrait être à l'origine d'une plus forte réponse à l'antibiotique en Rusitec que in vivo.

Le Rusitec est un modèle expérimental intéressant pour tester l'évolution qualitative des fermentations de la panse du Ruminant. Les résultats qu'il donne sur le plan quantitatif devront toutefois être interprétés avec prudence avant d'être extrapolés à l'animal.

Remerciements. - Les auteurs remercient C. Marpillat pour sa collaboration à l'expérimentation sur Rusitec.

Czerkawski J. W., Breckenridge G., 1977. Br. J. Nutr., 38, 371-384.

Durand M., 1982. Ann. Zootechn., 31, 47-46. 